\title{
Functional Regulation of the SLC26-Family Protein Prestin by Calcium/Calmodulin
}

\author{
Jacob Pearson Keller, ${ }^{1,2 \star}$ Kazuaki Homma, ${ }^{4,5 \star}$ Chongwen Duan, ${ }^{4}$ Jing Zheng, ${ }^{4,5}$ Mary Ann Cheatham, ${ }^{1,5}$ \\ and Peter Dallos ${ }^{1,3,5}$ \\ Departments of ${ }^{1}$ Communication Sciences and Disorders, ${ }^{2}$ Molecular Biosciences, ${ }^{3}$ Neurobiology, and ${ }^{4}$ Otolaryngology-Head and Neck Surgery, and ${ }^{5}$ The \\ Hugh Knowles Center for Clinical and Basic Science in Hearing and Its Disorders, Northwestern University, Evanston, Illinois 60208
}

The solute carrier gene family 26 (SLC26) encodes membrane proteins with diverse physiological roles but with the common feature of halide involvement. Here, we present bioinformatic and biochemical evidence that SLC26 proteins have intrinsically disordered regions (IDRs) in their C-terminal domains and that these regions contain calmodulin (CaM) binding sites. The veracity of these predictions and the functional consequences of CaM binding were examined in prestin, SLC26A5, as a model for the SLC26 family and as one of the most investigated and best understood members. We found that CaM binds directly to the IDR in the C-terminal domain of prestin in a calcium-obligate manner. Using both isolated murine outer hair cells $(\mathrm{OHCs})$ and a heterologous expression system, we also found that this calcium-obligate CaM binding shifts the operating point of the protein to more hyperpolarized potentials with consequent alteration of the function of the prestin. Because calcium is the main intracellular second messenger used by the efferent medial olivocochlear (MOC) pathway of the auditory system and CaM is abundant in OHCs, the CaM-prestin interaction may be involved in the MOC-mediated modulation of cochlear amplification. However, this regulatory mechanism is not likely to be restricted to cochlear OHCs, in light of both clear bioinformatic evidence and the fact that calcium and CaM are ubiquitous intracellular second messengers used by virtually all cell types. Hence, the calcium/CaM-dependent regulatory mechanism described herein is likely applicable to most, if not all, SLC26 paralogs.

Key words: calmodulin; disordered region; mice; prestin; SLC26 family

\section{Introduction}

Most solute carrier gene family 26 (SLC26) paralogs function as anion exchangers, whereas others are anion channels or even voltage-operated molecular motors (Alper and Sharma, 2013), with the common theme of halide involvement. Not surprisingly, mutations in SLC26 proteins are responsible for a variety of severe disorders, such as chondrodysplasias, Pendred's syndrome, congenital chloride diarrhea, deafness, and numerous others (for review, see Kere, 2006). Defining the regulatory mechanisms of SLC26 proteins is thus important for understanding both normal and pathological physiological processes. The relatively high sequence similarity with conserved tetrameric subunit stoichiom-

\footnotetext{
Received Sept. 19, 2013; revised Nov. 15, 2013; accepted Dec. 1, 2013.

Author contributions: J.P.K., K.H., and P.D. designed research; J.P.K., K.H., and C.D. performed research; J.Z. contributed unpublished reagents/analytic tools; J.P.K., K.H.,M.A.C., and P.D. analyzed data; J.P.K., K.H., M.A.C., and P.D. wrote the paper.

This work is supported by National Institutes of Health Grants DC00089 (P.D., M.A.C.) and DC010633 (J.Z.) and the Hugh Knowles Center. We acknowledge the Keck Biophysics Facility with Cancer Center Support Grant NCI CA060553, Dr. Predrag Radivojac for running CaM binding predictions, Dr. Julie Forman-Kay and Rebecca Keller for valuable help with this manuscript, Dr. Shmuel Muallem for providing SLC26A3 and A6 plasmids, Drs. Dhasakumar Navaratnam and Joseph Santos-Sacchi for providing the flipCBS prestin plasmid, and Mark F. Morel for his contribution for making $\Delta$ CBS construct.

*J.P.K. and K.H. contributed equally to this work.

The authors declare no competing financial interests.

Correspondence should be addressed to Peter Dallos, Department of Communication Sciences and Disorders,

Northwestern University, Evanston, IL 60208. E-mail: p-dallos@northwestern.edu.

J. P. Keller's present address: Janelia Farms Research Campus, Ashburn, VA 20147.

DOI:10.1523/JNEUROSCI.4020-13.2014

Copyright $\odot 2014$ the authors $\quad 0270-6474 / 14 / 341325-08 \$ 15.00 / 0$
}

etry between the SLC26 proteins (Hallworth et al., 2013) implies general functional and regulatory mechanisms shared among the family despite their distinct transport substrates. Consistent with this possibility, modulatory interactions with the cystic fibrosis transmembrane conductance regulator (CFTR) protein have been reported for several SLC26 paralogs (Ko et al., 2004; Chang et al., 2009; Homma et al., 2010; Rode et al., 2012). In addition, the fact that the functions of some SLC26 paralogs have been shown to be affected by manipulating intracellular calcium concentrations (Frolenkov et al., 2000; Loriol et al., 2008; Lamprecht et al., 2009; Keller et al., 2013) further points to the presence of a generalized regulatory mechanism shared among the family.

Prestin (SLC26A5; Zheng et al., 2000) is one of the best characterized members in the SLC26 family in terms of both structure (Pasqualetto et al., 2010) and function (Ashmore, 2008). Although non-mammalian prestin homologs are transporters, mammalian prestin homologs are expressed exclusively in outer hair cells (OHCs) of the cochlea, in which they act both as voltage-operated motors (Zheng et al., 2000) and weak transporters (Mistrík et al., 2012). The resulting voltage-dependent somatic motility, often referred to as OHC electromotility (Brownell et al., 1985), is thought to drive mechanical force feedback in the cochlea, thereby enhancing both the sensitivity and frequency selectivity of the mammalian ear (Dallos, 1992). A potentially related property, $\mathrm{OHC}$ axial stiffness, is both voltage dependent (He and Dallos, 1999) and is reduced by acetylcholine (ACh)-elicited increase in intracellular calcium, one of the major features of suppressive feedback, cochlear gain regulation 
via the medial olivocochlear (MOC) efferents (Dallos et al., 1997). Evidence suggests that prestin significantly contributes to the global $\mathrm{OHC}$ axial stiffness and that ACh affects both prestin and other lateral wall stiffness components (He et al., 2003). A previous study reported alteration of $\mathrm{OHC}$ motor properties subsequent to addition of inhibitors of the ubiquitous and well characterized calciumresponsive protein calmodulin (CaM; Frolenkov et al., 2000), which is highly expressed in OHCs (Slepecky and Ulfendahl, 1993). In light of this knowledge, along with the rigor with which the function and physiology of prestin has been heretofore characterized, we chose prestin as a model SLC26 protein through which the nature of CaMbased regulatory mechanisms of SLC26 proteins might be explored.

Herein, we present evidence that $\mathrm{CaM}$ directly binds to the C-terminal domain of prestin in a calcium-obligate manner and that this binding significantly hyperpolarizes the voltageoperating point $\left(V_{\mathrm{pk}}\right)$ of prestin, which has the potential to modulate $\mathrm{OHC}$ axial stiffness attributable to variations in the state of individual prestin molecules (He and Dallos, 1999; He et al., 2003). Moreover, we present clear bioinformatic evidence that this CaM-dependent regulatory mechanism can be extrapolated to all SLC26 proteins.

\section{Materials and Methods}

CaM binding assays. Recombinant human CaM was prepared as described previously (Ikebe et al., 1998). A 30 aa peptide corresponding to gerbil prestin K557-N586 (KRKTGVNPALIMGARRKAMRKYAKEVGNAN) was synthesized with an $\mathrm{N}$-terminal fluorescein label and was dissolved at $5 \mathrm{~mm}$ in water before storage at $-80^{\circ} \mathrm{C}$. Size exclusion chromatography (SEC) experiments were run using a Superdex 200 10/300 GL column on an AKTA purifier system in running buffer containing $150 \mathrm{~mm} \mathrm{NaCl}$ and 10 mM Tris-HEPES, pH 7.5, supplemented with either $5 \mathrm{~mm} \mathrm{CaCl}_{2}$ or $5 \mathrm{~mm}$ $\mathrm{Na}-\mathrm{EDTA}$ as dictated by the experiment. Injected samples were prepared by premixing $10 \mu \mathrm{l}$ of CaM stock at $20 \mathrm{mg} / \mathrm{ml}$ in $50 \mathrm{mM} \mathrm{NaCl}$ and $10 \mathrm{mM}$ Tris-HEPES, pH 7.5, with $10 \mu \mathrm{l}$ of running buffer or peptide stock solution (water-dissolved peptide stock solution diluted in running buffer to $10 \mathrm{mg} / \mathrm{ml}$ ), supplemented to $5 \mathrm{~mm} \mathrm{CaCl}_{2}$ or Na-EDTA and incubated at room temperature for $\sim 20 \mathrm{~min}$. Fractions $(0.5 \mathrm{ml})$ were collected and subsequently analyzed on a fluorescence plate reader (BioTek) for measurement of fluorescence intensity and polarization signals. Fluorescence polarization experiments to determine the affinity of the CaM-peptide complex were performed as follows: 1:2 serial dilutions of CaM at 37.5 $\mathrm{mg} / \mathrm{ml}$ in $50 \mathrm{~mm} \mathrm{NaCl}$ and $25 \mathrm{~mm}$ Tris-Cl, $\mathrm{pH} 8.0$, were made into measurement buffer containing $10 \mathrm{~nm}$ peptide, $20 \mathrm{~mm}$ Tris-HEPES, $\mathrm{pH}$ 8.0, $50 \mathrm{~mm} \mathrm{NaCl}, 0.1 \%$ Tween 20 , and $2 \mathrm{~mm} \mathrm{CaCl}_{2}$. Polarization values were measured on a Panvera Beacon 2000 instrument in triplicate. Immediately after these measurements were made, Na-EDTA, $\mathrm{pH}$ 8.0, was added to the samples to $5 \mathrm{~mm}$, and the fractions were remeasured. Resulting curves were plotted and fitted in GraphPad Prism using the Hill equation.

Electrophysiology. Adolescent FVB mice of both sexes (P37-P47) were killed with euthasol (200 mg/kg, i.p.), and OHCs from the apical region of the cochlea were isolated as described previously (Cheatham et al., 2005). Wild-type (WT) and mutated gerbil recombinant prestin constructs were cloned into a pEGFP-N2 vector and expressed in HEK293T cells as described previously (Zheng et al., 2005; Homma et al., 2010). Whole-cell nonlinear capacitance (NLC) recordings were performed using the Axopatch 200A amplifier (Molecular Devices). An intracellular solution contained the following (in mM): $150 \mathrm{CsCl}, 2 \mathrm{MgCl}_{2}, 0.1 \mathrm{CaCl}_{2}$ (or 10 EGTA), and 10 (or 20) HEPES, pH 7.4. An extracellular solution contained the following (in mM): $150 \mathrm{NaCl}, 2 \mathrm{MgCl}_{2}, 2 \mathrm{CoCl}_{2}$, and 10 HEPES, pH 7.4. Osmolarity was adjusted to $340 \mathrm{mmol} / \mathrm{kg}$ with glucose. Intracellular pressure was kept at $0 \mathrm{mmHg}$. Data were collected by jClamp (SciSoft) using a fast Fourier transform-based admittance analysis to determine cell membrane capacitance $\left(C_{\mathrm{m}}\right)$ (Santos-Sacchi et al., 1998). $C_{\mathrm{m}}$ data were analyzed using the following two-state Boltzmann equation:

$$
C_{\mathrm{m}}=\frac{\alpha Q_{\mathrm{max}} \exp \left[\alpha\left(V_{\mathrm{m}}-V_{\mathrm{pk}}\right)\right]}{\left\{1+\exp \left[\alpha\left(V_{\mathrm{m}}-V_{\mathrm{pk}}\right)\right]\right\}^{2}}+C_{\text {lin }},
$$

where $\alpha$ is the slope factor of the voltage dependence of charge transfer, $Q_{\max }$ is the maximum charge transfer, $V_{\mathrm{m}}$ is the membrane potential, $V_{\mathrm{pk}}$ is the voltage at which the maximum charge movement per voltage is attained, and $C_{\text {lin }}$ is the linear capacitance (Homma et al., 2010, 2013; Homma and Dallos, 2011).

\section{Results}

The SLC26 proteins contain $\sim 750$ aa divided into an intracellular $\mathrm{N}$-terminal segment ( $\sim 100$ residues), an intramembranous region ( $\sim 400$ residues) that is predicted to contain $10-12$ transmembrane helices, and an intracellular C-terminal domain ( $\sim 250$ residues) that contains a conserved sulfate transport and anti-sigma factor antagonist (STAS) domain (Sharma et al., 2011). Based in part on a previous study of prestin (SLC26A5; Frolenkov et al., 2000), we considered whether there might be a CaM-mediated regulatory mechanism common to all SLC26 proteins. As a first step, we explored the general sequence properties of SLC26 proteins using several bioinformatic approaches. The first of these was prediction of intrinsically disordered regions (IDRs), which revealed an area of high IDR likelihood in most of the STAS domains of the SLC26 proteins (Fig. 1 A, C, plot labeled IDR). Difficulty in crystallizing the STAS domains of SLC26 proteins, including that of prestin (Babu et al., 2010; Pasqualetto et al., 2010), can be at least partially explained by the presence of such high IDR likelihood regions, with IDR truncation apparently a sine qua non for success. Because CaM binding sites are often located in IDRs (Uversky and Dunker, 2010), these regions were of particular interest. To test these predictions empirically for the presence of IDRs, we ran SDS-PAGE gels of purified STAS constructs searching for one of the hallmarks of IDRs: aberrantly slow electrophoretic mobility (Radivojac et al., 2007). This aberrant mobility ( $\sim 9 \mathrm{kDa}$ upward shift between the calculated vs apparent molecular masses) was indeed found in all constructs tested (A3, A5, and A6) (Fig. 1B) and was corroborated by similar mobility shifts in other orthologs (Dorwart et al., 2008; Pasqualetto et al., 2008). Subsequent limited trypsinolysis experiments on the prestin-STAS construct showed that the three sites most susceptible to trypsin digestion were located in the predicted IDR (K580, K606, and K622 in gerbil prestin), further corroborating its flexible nature and the amino acids potentially involved at the interface between prestin and CaM. Based on these considerations, the presence of IDRs in the C-terminal domains of the SLC26 proteins was highly likely.

Because CaM binding sites (CBS) are frequently located in IDRs (Radivojac et al., 2006, 2007), we used a well characterized CBS prediction program (Radivojac et al., 2006) to assess the likelihood of CBSs existing therein and found in each paralog a region of high CBS likelihood within the aforementioned IDR (Fig. $1 A, C$, plot labeled CaM). Because the scores were quite high across all of the homologs, this strongly suggested physiological binding of CaM. Furthermore, because the sites were located in IDRs, the CBS would be accessible for binding to CaM. We consider in more depth the statistics of the predictor and their ramifications in the discussion section below. These data together indicated that all SLC26 homologs tested have IDRs and bind CaM.

To demonstrate empirically CaM binding and its functional consequences, we chose to focus on prestin (SLC26A5) as a model for the SLC26 family because it is the best characterized member in terms of structure (Pasqualetto et al., 2010) and func- 
A

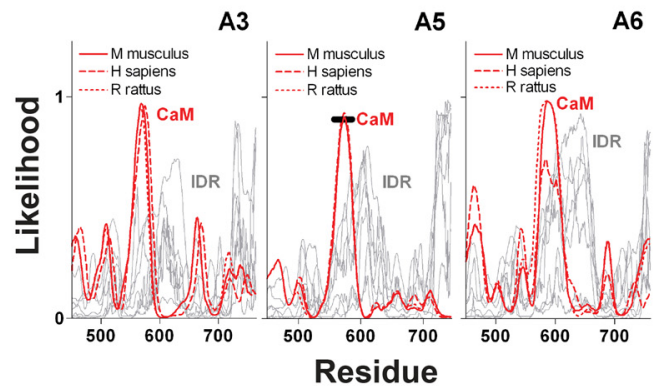

C
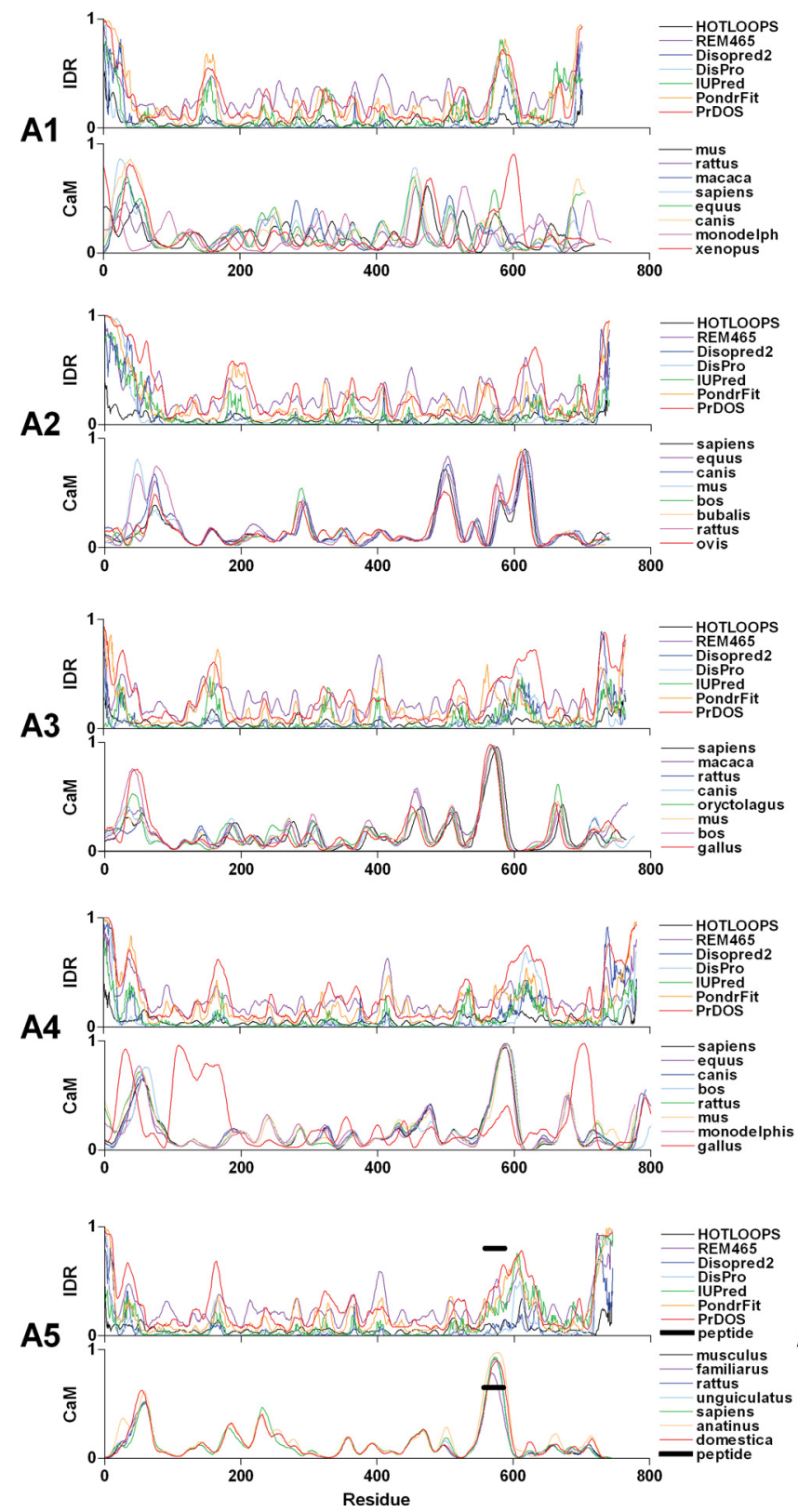

B

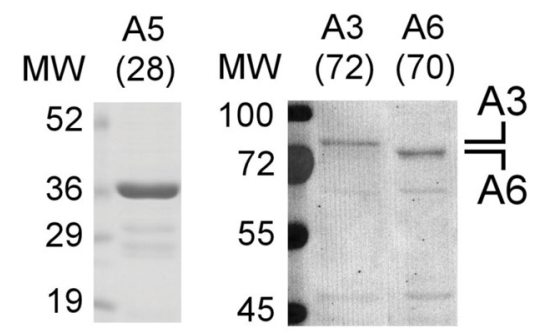

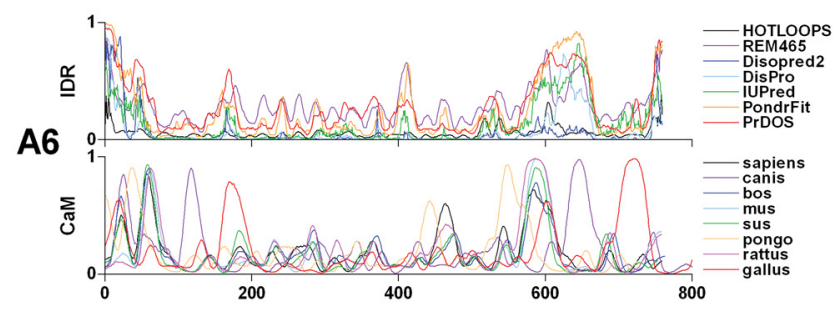
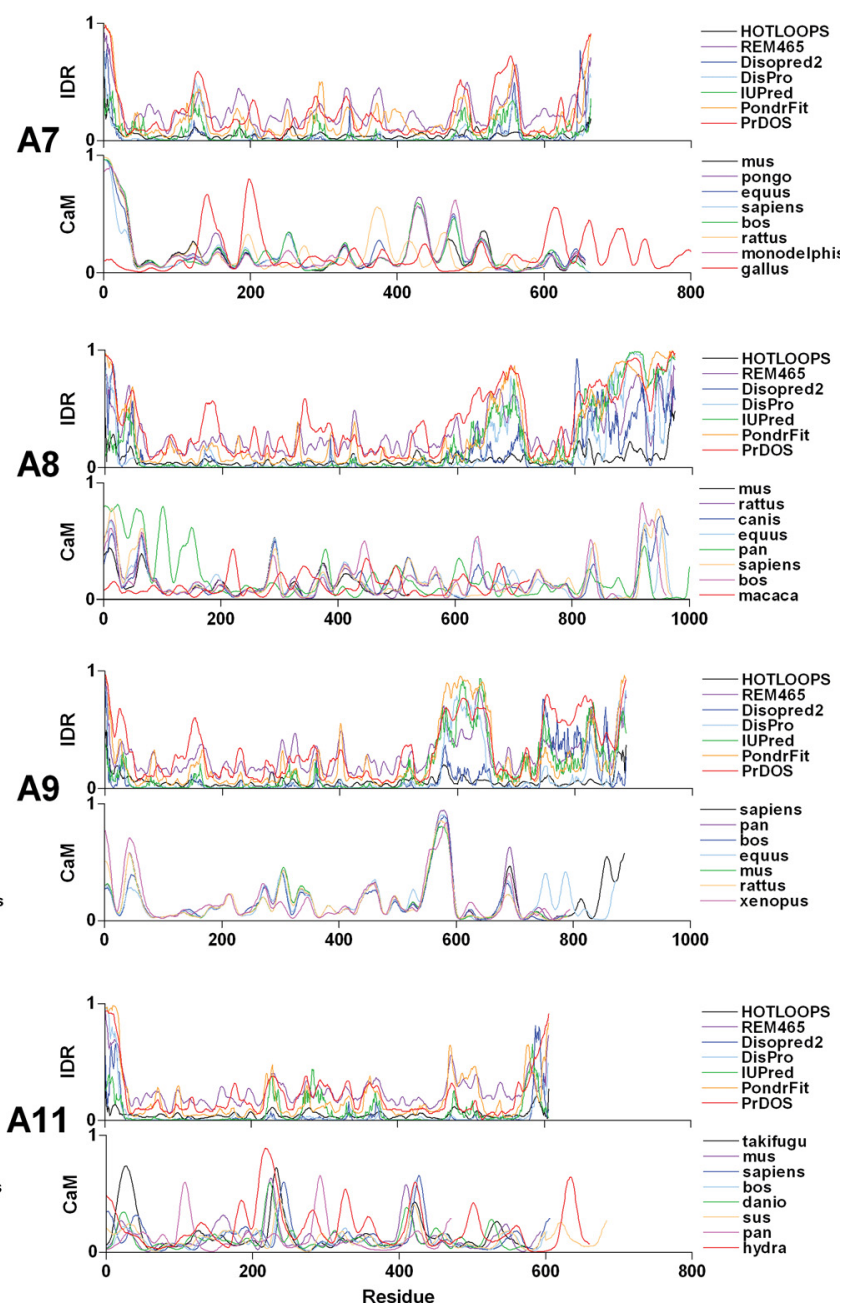

Figure 1. Bioinformatic predictions of IDRs and CBSs. A, Scores for IDR (gray) and CaM binding (red) in the C-terminal domains of SLC26A3; A5, and A6 are plotted as a function of residue number. In traces for A5 (prestin), a horizontal bar indicate the region corresponding to the synthesized peptide used in Figure 2. $\boldsymbol{B}$, Aberrant SDS-PAGE mobility of purified SLC26 STAS domains. Coomassie-stained SDS-PAGE gel of purified STAS constructs from A3, A5, and A6. Maltose binding protein ( $42.5 \mathrm{kDa}$ ) was attached to the N termini of the A3 and A6 constructs. Standard molecular weight (MW) markers are shown on the left in kilodaltons, and calculated molecular weight of each STAS construct is shown in parentheses at the top. $C$, A complete set of bioinformatic predictions of IDRs and CBSs for all SLC26 paralogs. In traces for A5 (prestin), horizontal bars indicate the region corresponding to the synthesized peptide used in Figure 2 . As in $\boldsymbol{A}$, predictor scores are plotted as a function of residue number for each homolog (e.g., A3 represents SLC26A3). Plots labeled "IDR" show predictions of intrinsic disorder from seven different programs, H0TL00PS, REM465, Disopred2, DisPro, IUPred, PondrFit, and PrD0S (indicated on the right of the panels; Linding et al., 2003; Ward et al., 2004; Cheng et al., 2005; Dosztányi et al., 2005; (Figure legend continues.) 

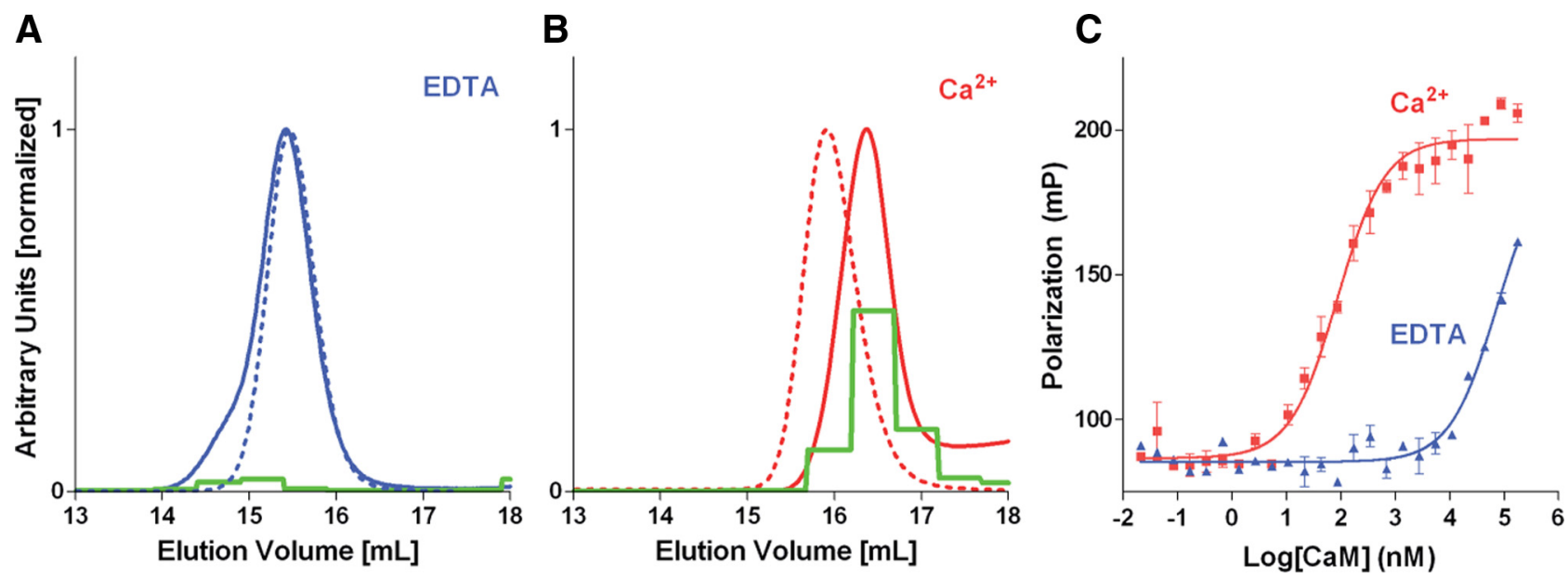

Figure 2. Biochemical assays of the CaM-SLC26 interaction. $A, B$, SEC of CaM in the presence (blue and red solid lines) and absence (blue and red broken lines) of fluorescein-labeled prestin peptide under EDTA $(\boldsymbol{A})$ or $\mathrm{CaCl}_{2}(\boldsymbol{B})$ conditions. Fluorescence intensities are shown with solid green lines. $\boldsymbol{C}$, Fluorescence polarization assay of CaM versus labeled prestin peptide in the presence (red) or absence (blue) of calcium. Error bars represent SDs of three measurements, with fits to the Hill equation superimposed on data points.

tion (Ashmore, 2008). Using a 30 aa fluorescein-tagged peptide corresponding to the predicted CBS in prestin (Fig. $1 A, C$, horizontal bars in prestin IDR and CaM plots), we performed SEC on combinations of calcium, CaM, and peptide to determine whether-and under which conditions - a CaM-peptide complex would form (Fig. 2A,B). Coelution of fluorescein-peptide signal (Fig. $2 A, B$, green lines) with CaM occurred in the presence of calcium (Fig. $2 B$, solid red trace) but not appreciably in its absence (Fig. 2A, solid blue trace), revealing an unambiguous calcium-obligate interaction. However, in the course of analyzing these data, we noticed a surprising phenomenon brought about by the presence of peptide: whereas in the EDTA condition the small peptide-containing shoulder (Fig. $2 A$, solid blue line) was unremarkably of apparently higher molecular weight than apoCaM (Fig. $2 A$, broken blue line) in the calcium condition, the single peak corresponding to the peptide-CaM complex (Fig. $2 B$, solid red line) was robustly and repeatedly shifted in the direction of lower apparent molecular weight compared with CaM alone (Fig. $2 B$, broken red line). Although unexpected at first, the result is readily understood considering the phenomenon, documented in the literature, of other CaM-peptide complexes having smaller hydrodynamic radii than unbound CaM, despite their moderately $(\sim 3 \mathrm{kDa})$ higher molecular weight. Although molecular weight and hydrodynamic radius generally covary, CaM and its peptide complexes violate this rule because of the extended conformation of unbound CaM versus the more compact conformations of CaM-peptide complexes. Because SEC effectively measures not molecular weight but hydrodynamic radius, the initially surprising result provided support for the prestin-CaM interaction being similar to the canonical complexes rather than the more recently elucidated unconventional examples. Ironically, in light of this explanation, the complex observed in the EDTA condition now required explanation: this complex must have a larger hydrodynamic radius than when CaM is unbound, consistent with an open, extended peptide-bound conformation. However, because only a small fraction of CaM eluted as a com-

\section{$\leftarrow$}

(Figure legend continued.) Ishida and Kinoshita, 2007; Xue et al., 2010), run on the SLC26 ortholog from Homo sapiens. Plots labeled "CaM" show output from a well characterized CaM binding prediction algorithm (Radivojac et al., 2006) run on orthologs from eight species also indicated on the right of the panels. plex despite the presence of excess peptide in the sample, we evaluated the calcium-independent CaM-peptide interaction to be of low affinity (quantified below) and therefore possibly an experimental artifact of dubious physiological relevance. To explore this further, the affinities of the interaction were subsequently determined by a fluorescence polarization assay (Fig. 2C) to be $86.5 \mathrm{nM}\left(\mathrm{CaCl}_{2}\right)$ and $73.4 \mu \mathrm{M}$ (EDTA), i.e., an $\sim 1000$-fold difference. Similarly sized fluorescein-tagged control peptides showed essentially no binding $\left(K_{\mathrm{d}}>100 \mu \mathrm{M}\right)$ regardless of calcium. Both affinity and calcium responsiveness were consistent with values reported for verified physiological CaM-peptide interactions, with a mean $K_{\mathrm{d}}$ value at $\sim 130 \mathrm{~nm}$ (O'Neil and DeGrado, 1990). Hence, the bioinformatics predictions were corroborated empirically, and the interaction was shown to be of high affinity and to require calcium.

We also considered the ramifications of the CaM-prestin interaction in cochlear physiology and endeavored to measure the effects of the interaction in conditions more closely resembling those in vivo. ACh is used by the MOC efferents to modulate cochlear amplification (Eybalin, 1993). It was reported previously that ACh induces a small but statistically significant hyperpolarizing shift $(-4 \mathrm{mV})$ in the $V_{\mathrm{pk}}$ of prestin (Frolenkov et al., 2000), as well as a reduction in the axial stiffness of the OHC (Dallos et al., 1997) through elevations in intracellular calcium. We speculated that the direct prestin-CaM interaction demonstrated above might be at least partially responsible for these changes. Because the aforementioned calcium effect on $V_{\mathrm{pk}}$ was seen even under weak calcium-chelating conditions $(0.5 \mathrm{~mm}$ EGTA; Frolenkov et al., 2000), removal of EGTA might unmask an even larger effect. To this end, we measured whole-cell NLC in mouse $\mathrm{OHCs}$ as a function of intracellular calcium (Fig. 3A-C,E). As predicted, large hyperpolarizing $V_{\mathrm{pk}}$ shifts $(-35 \pm 19 \mathrm{mV})$ were observed with intracellularly applied calcium. These hyperpolarizing $V_{\mathrm{pk}}$ shifts were reversed during local perfusion of the CaM inhibitor trifluoperazine (TFP), indicating that the $V_{\mathrm{pk}}$ shift was, in fact, both dependent on CaM and readily reversible (Fig. $3 D, E)$. As control recordings to assess a potential effect of extracellular calcium on our whole-cell NLC recordings, we compared NLCs measured in the presence and absence of extracellular calcium. The $\alpha, V_{\mathrm{pk}}$, and charge density values determined in the presence $\left(2 \mathrm{mM} \mathrm{CaCl}_{2}\right)$ and absence $\left(2 \mathrm{mM} \mathrm{CoCl}_{2}\right)$ of extracellular calcium were statistically insignificant $\left[0.032 \pm 0.003 \mathrm{mV}^{-1}\right.$ 
A
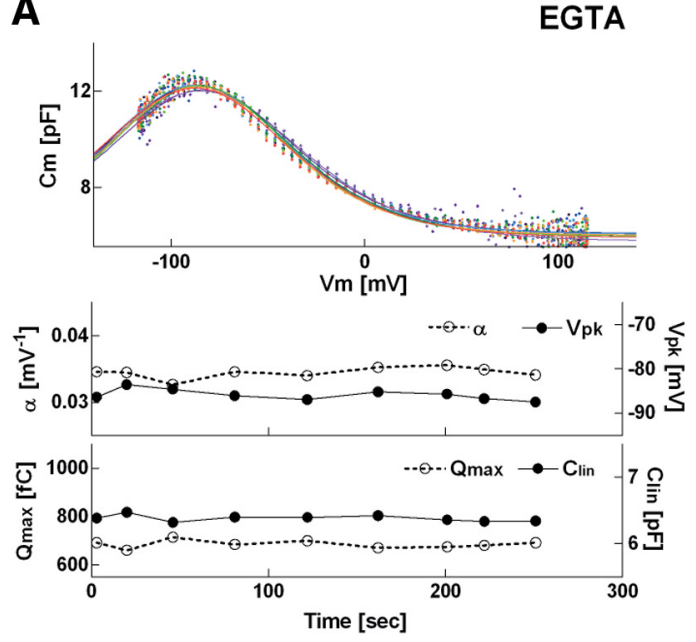

B
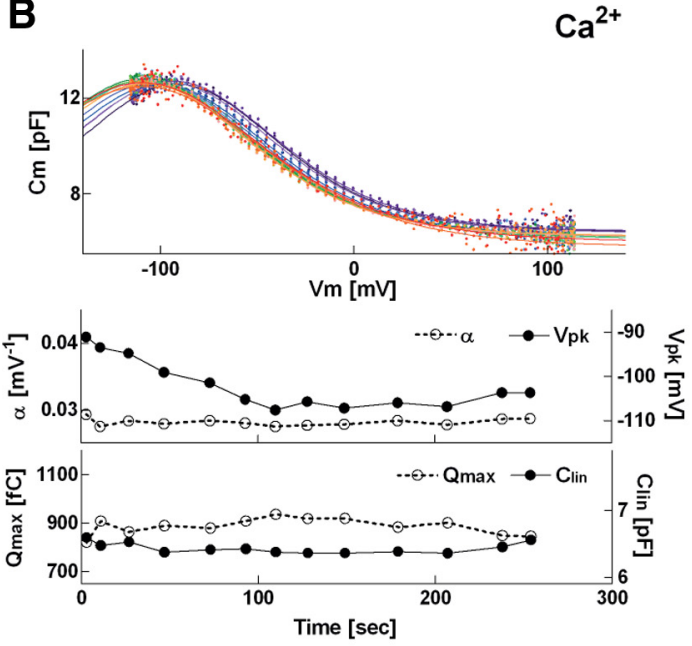

C

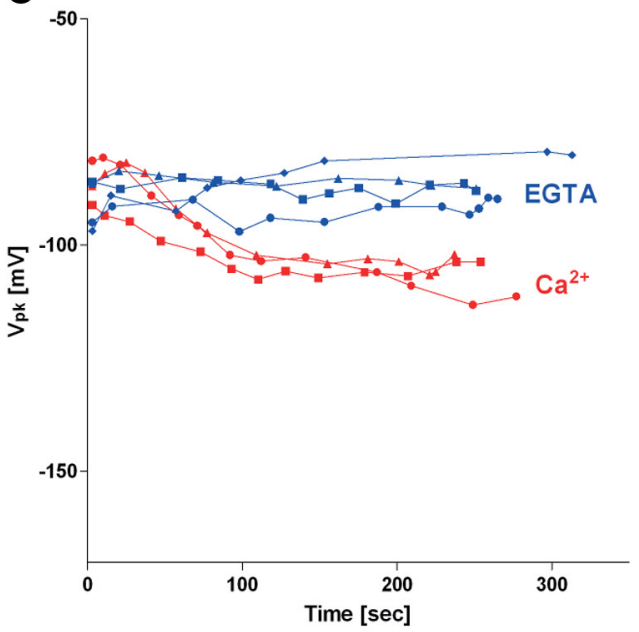

D

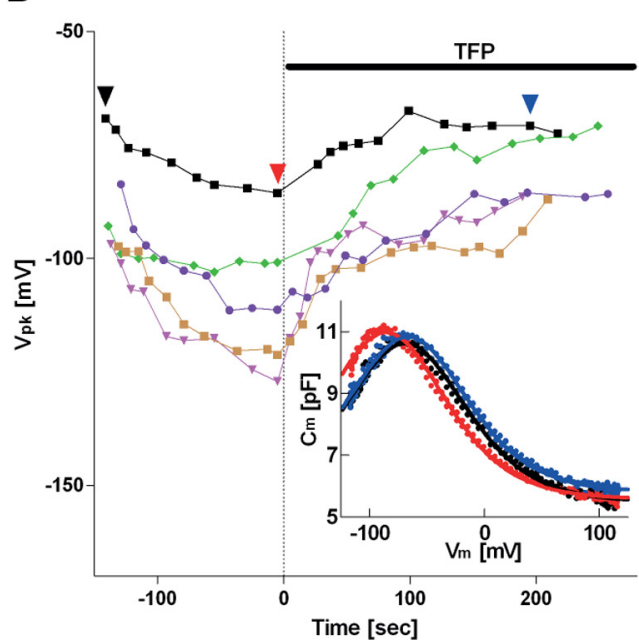

$\mathbf{E}$

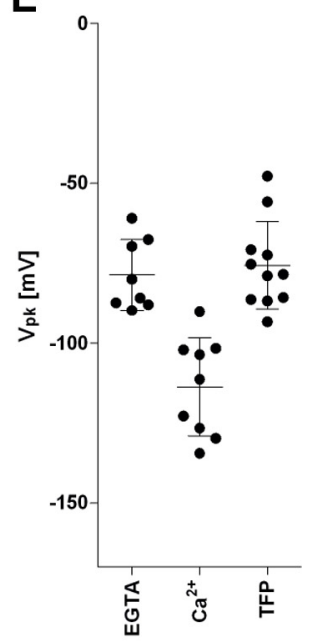

Figure 3. Whole-cell NLC recordings in isolated murine OHCS. $\boldsymbol{A}, \boldsymbol{B}$, Examples of time-dependent NLCs measured under the EGTA $(\boldsymbol{A})$ and calcium (B) conditions (top panels). Different colors indicate NLC measurements at different time points. $V_{\mathrm{pk}}$ and $\alpha$ values (middle panels) and $Q_{\max }$ and $C_{\text {lin }}$ values (bottom panels) were determined for each NLC curve and plotted against time. $C$, A summary of the time course of $V_{\text {pk }}$ under EGTA (blue) and calcium $\left(\mathrm{Ca}^{2+}{ }^{2+}\right.$, red) conditions. Different symbols represent different OHCs. D, After $\sim 2$ min in the calcium condition, TFP (50 $\left.\mu \mathrm{M}\right)$ was locally perfused at the time indicated by the vertical broken line. The horizontal black bar indicates duration of the TFP perfusion. The inset shows examples of NLCs determined at different time points, indicated by arrowheads, during a time-dependent NLC recording (black squares). The colors of the arrowheads correspond to the same-colored NLC curves. $E, V_{\text {pk }}$ values at the end of each time course are summarized for comparison. ANOVA and subsequent Tukey's analysis found a statistically significant difference between EGTA versus $\mathrm{Ca}^{2+}(p<0.001)$ and between TFP versus $\mathrm{Ca}^{2+}$ conditions $(p<0.001)$ but not between EGTA versus TFP conditions $(p>0.05)$. No statistically significant difference was found for the other NLC parameters, $\alpha$ and $Q_{\max }$ (ANOVA, $\left.p>0.05\right)$. The error bars represent \pm SD.

$\left(n=5, \mathrm{Ca}^{2+}\right)$ vs $0.028 \pm 0.004 \mathrm{mV}^{-1}\left(n=8, \mathrm{Co}^{2+} ; p=0.11\right)$, $-82 \pm 9 \mathrm{mV}\left(n=5, \mathrm{Ca}^{2+}\right) \mathrm{vs}-79 \pm 11 \mathrm{mV}\left(n=8, \mathrm{Co}^{2+} ; p=\right.$ $0.55)$, and $145 \pm 16 \mathrm{fC} / \mathrm{pF}\left(n=5, \mathrm{Ca}^{2+}\right)$ vs $150 \pm 19 \mathrm{fC} / \mathrm{pF}(n=$ $\left.\left.8, \mathrm{Co}^{2+} ; p=0.66\right)\right]$, confirming that our NLC recordings were not affected by the nominal calcium-free extracellular solution used in this study (for the ionic composition; see Materials and Methods).

To address the possibility of CaM acting indirectly or upstream of prestin, as well as to confirm involvement of the IDR within prestin, we measured the calcium-induced shift in two different CBS-altered prestin constructs (Fig. 4) transfected into HEK293T cells (Fig. 5), both of which completely abrogate bioinformatic CBS predictions (Fig. 4). After confirming the presence of the calcium-induced $V_{\mathrm{pk}}$ shift in HEK293T cells transfected with WT prestin (Fig. $5 A, B$ ), as well as its TFP sensitivity (Fig. $5 C, D)$, we measured the effect in a CBS-deleted construct $(\Delta 571-$ 635 , referred to as $\Delta \mathrm{CBS}$ ) and found that this mutation completely abolished the calcium-dependent shift in $V_{\mathrm{pk}}$ (Fig. $5 E, F$ ), mimicking the effects of TFP on WT prestin. We cross-validated this result with an additional CaM binding-deficient mutant in which six of the basic residues (R571, R572, K573, R576, K577, and $\mathrm{K} 580$ ) in the CBS were all mutated to aspartates to abrogate CaM binding ("flipCBS"; Bai et al., 2006) and found that the $V_{\mathrm{pk}}$ of this mutant also does not respond to intracellularly applied calcium (Fig. 5G,H). These electrophysiological experiments, together with the bioinformatic and biochemical experiments detailed above, demonstrate that direct binding of CaM to the IDR in the intracellular C-terminal STAS domain of prestin causes hyperpolarizing shifts in the voltage dependence $\left(V_{\mathrm{pk}}\right)$ of prestin and presumably affects other SLC26 proteins in similar ways, although perhaps with different consequences, i.e., differences in transport activity rather than electromotility.

\section{Discussion}

It is important first to consider the quantitative aspects and ramifications of the bioinformatic predictions of CBSs both with re- 


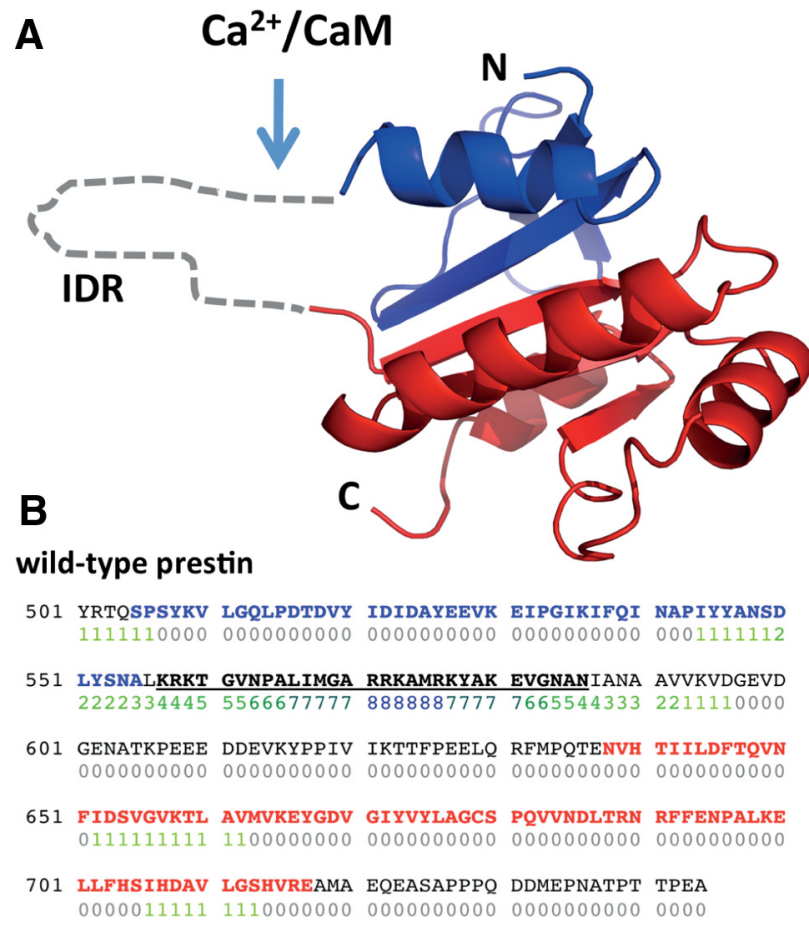

C

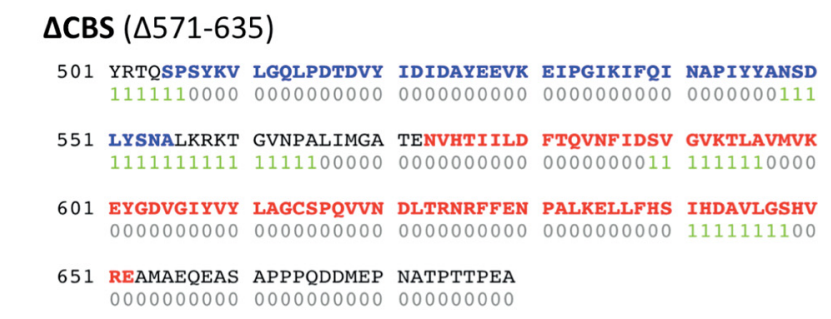

Figure 4. $\quad \boldsymbol{A}, \boldsymbol{B}, \mathrm{CaM}$ binding site in the $\mathrm{C}$-terminal domain of prestin. The structure of the $\mathrm{C}$-terminal domain of rat prestin (Pasqualetto et al., 2010) is shown $(\boldsymbol{A})$ ). The structures shown in blue and red correspond to the same-colored amino acid sequences of the C-terminal domain of gerbil prestin $(\boldsymbol{B})$. A predicted IDR is drawn with a broken gray line. The numbers below the amino acid sequence indicate the score for CaM binding (scaled from 0 to 9) determined by a CaM binding site prediction program (Radivojac et al., 2006). CaM binding to this high CaM binding likelihood region was demonstrated in this study (Fig. 2) using a synthesized peptide whose sequence corresponds to that shown in bold black with underline. $C, D$, Two mutated prestin constructs used for assessing functional consequence of direct CaM binding to prestin. The sequences shown in blue and red correspond to the structures shown with the same colors in $A$. In $\triangle C B S(C)$, most of the IDR region containing the CaM binding site was deleted. In flipCBS ( $\boldsymbol{D}$, also known as "cluster b"; Bai et al., 2006), the six basic residues residing in the CaM binding region were mutated to the acidic residue asparatate to abrogate CaM binding (highlighted in yellow). The score for CaM binding was recalculated for each construct (shown below the amino acid sequence as in $\boldsymbol{B}$ ). Only $\mathrm{C}$-terminal domain amino acid sequences are shown for both constructs.

gard to prestin as well as the rest of the SLC26 protein family (Fig. 1). Although some bioinformatics programs are poorly characterized in terms of the meaning of a given score, this particular predictor (Radivojac et al., 2006) has been quantified rigorously by using training and test sets of the hundreds of known CBSs, allowing the output scores to be cast reliably as either sensitivities and specificities or, perhaps more usefully, Bayesian likelihood ratios (LRs) (Fig. 6A). For example, with this predictor, scores 0.92 - seen in many of the SLC26 paralogs (Fig. $1 A, C)$ correspond to LRs $>30$. To put this number in context, the average LR of $\sim 50$ common clinical tests was 16.8 , with a median of 6.8 (reported at http://www.med. unc.edu/medicine/edursrc/lrtest.htm). Using the Bayesian formalism, one can use this LR value mathematically to convert prior probabilities into posterior probabilities (Fig. 6B). An approach to generalization of the current bioinformatic results then might be to determine what prior probability would lead to a posterior probability of $>0.95$ (analogous to $p<0.05$ ), given the observed predictor scores, and then evaluate how this number corresponds to
A
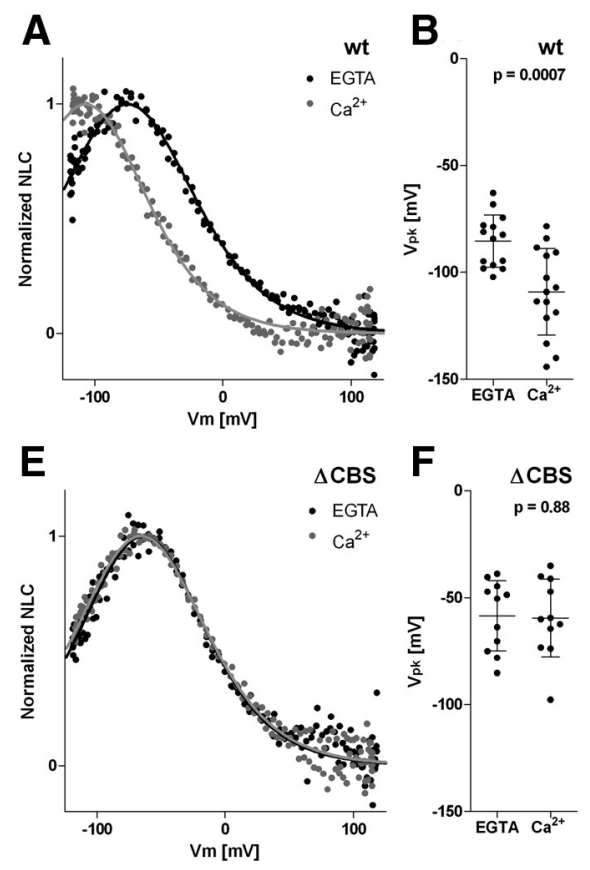
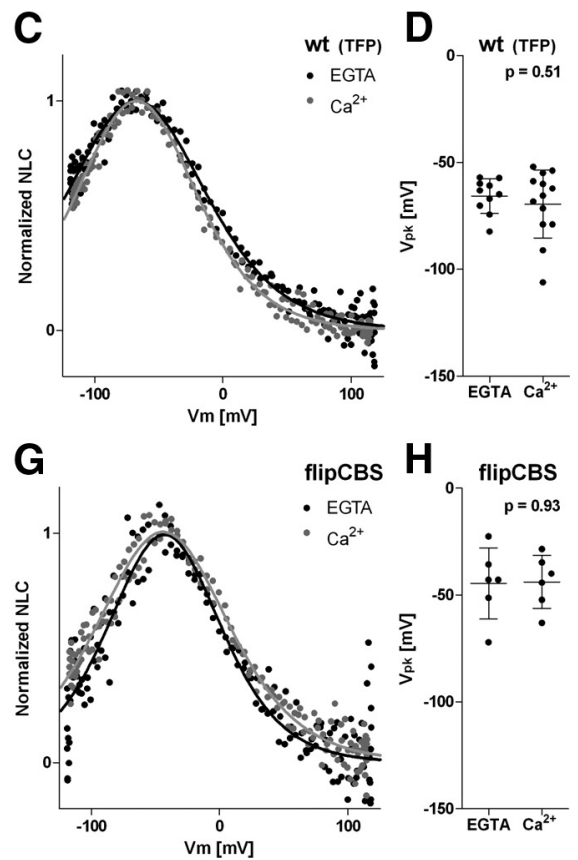

Figure 5. Whole-cell NLC recordings in HEK293T cells heterologously expressing WT $(\boldsymbol{A}-\boldsymbol{D}), \Delta C B S(\boldsymbol{E}, \boldsymbol{F})$, and flipCBS $(\boldsymbol{G}, \boldsymbol{H})$. NLC was measured in EGTA and calcium ( $\left(\mathrm{Ca}^{2+}\right)$ conditions. Representative NLC curves are shown for each construct $(A, C, E, G)$, and $V_{\text {pk }}$ values are summarized for comparison $(\boldsymbol{B}, \boldsymbol{D}, \boldsymbol{F}, \boldsymbol{H})$. The $p$ values determined by the Student's $t$ test are shown in each graph. No statistically significant difference was found between EGTA versus calcium conditions for the other NLC parameters, $\alpha$ and $Q_{\max }$ $(p>0.05)$. The error bars represent \pm 1 SD. 


\section{A Likelihood Ratio Vs. Predictor Score}

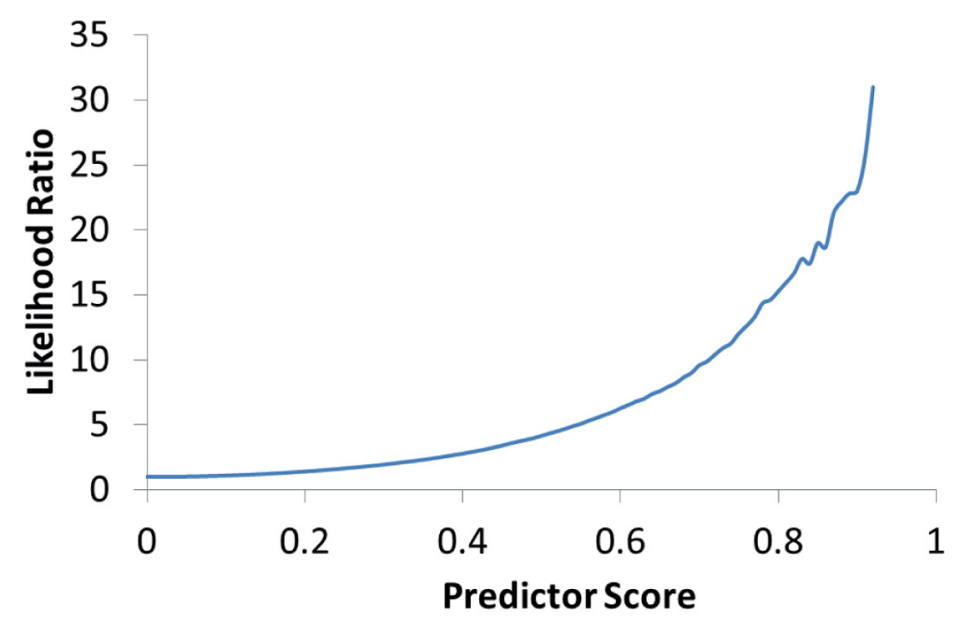

\section{B \\ Posterior Vs. Prior Probability at Various Predictor Scores}

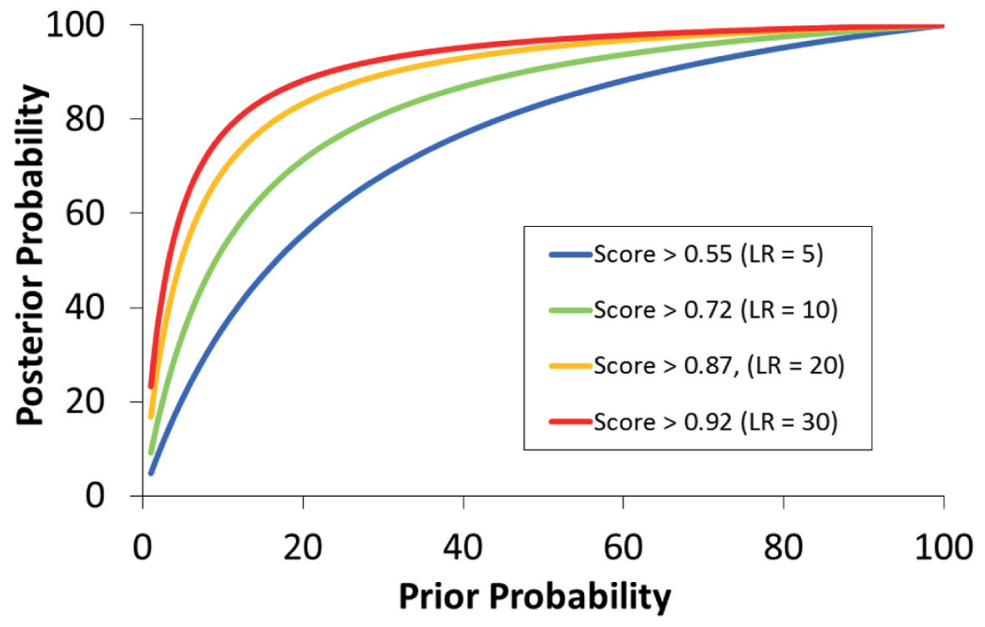

Figure 6. Bayesian statistics of the CaM predictor. Scores from the predictor are associated with sensitivities and specificities by using known CaM targets not included in the training set of the predictor. These can in turn be converted into $L R s(\boldsymbol{A})$. Using LRs, prior probabilities can be mathematically converted into posterior probabilities $(\boldsymbol{B})$. Conversion curves are shown for several levels of $L R$.

been shown previously to be functionally modulated by intracellular calcium [SLC26A3 (Lamprecht et al., 2009), SLC26A5 (the present study and Frolenkov et al., 2000; He et al., 2003), and SLC26A9 (Loriol et al., 2008)]. We suggest that there is a very high likelihood that at least the high-scoring paralogs in the SLC26 family share the prestin attribute of calcium/ CaM-based functional modulation.

The functional data presented above indicate that the voltage-dependent energy states of prestin, as represented by $V_{\mathrm{pk}}$, are perturbed by the binding of CaM. In light of what is known about cochlear and $\mathrm{OHC}$ physiology, the ramifications and mechanism of the interaction are straightforward. Because it has been shown that voltage-dependent $\mathrm{OHC}$ stiffness is intimately related to the $V_{\mathrm{pk}}$ of prestin, it follows that cochlear mechanics and, hence, cochlear amplification, would be affected by CaM-prestin binding. Furthermore, because MOC activity both elevates $\mathrm{OHC}$ calcium as well as suppresses cochlear amplification, it appears likely that the CaM-prestin interaction plays a role in modulation of cochlear amplification via MOC effects.

However, what may remain unclear is how CaM-induced changes in the $V_{\mathrm{pk}}$ of prestin correspond to aspects of molecular function in other SLC26 homologs. Although there is a possibility that CaM exerts its effects on SLC26 proteins in homolog-specific ways, we propose the formulation of a general mechanism of CaM-induced inhibition involving perturbations of relative energies of transporter states or analogous conformational states, as follows. Although the degree to which the voltage-dependent states of prestin correspond to vestigial transporter

estimates of actual prior probabilities. In the case of most of the SLC26 family, this requisite prior probability is 0.38 . As a minimal baseline, assuming there are at least 200 CaM targets in the proteome (some reports suggest many more than that; Shen et al., 2005 ) and a proteome size of 20,000 proteins, the prior probability for any protein would be 0.01 , with the corresponding posterior probability of 0.23 given scores equivalent to those observed for the SLC26 proteins. Starting first with prestin, for which the current study provides strong empirical evidence, the prior probability would easily surpass 0.38 , leading to a posterior probability of $\gg 0.95$, and this would propagate to other prestin orthologs because of their very high sequence similarity.

Although extrapolation to the SLC26 paralogs of prestin might be less straightforward, there are nevertheless some considerations that may make the case stronger. First, the entire family shares similar sequence, predicted secondary structure, and predicted topology, suggesting a similarity in architecture that may parallel a similarity in functional mechanism. Second, there are many functional studies that show similarity in chloride involvement, CFTR binding, and epithelial targeting. Third, and perhaps most saliently, several of the non-prestin paralogs have states remains controversial, all agree that there is some relationship, albeit to varying degrees under different conditions. Because there is some degree of correspondence then, the NLC of prestin may be considered a reflection of the relative energy of the vestigial transporter states of prestin, with $V_{\mathrm{pk}}$ representing the transmembrane voltage at which the energies of the transporter states are equal. It then follows that CaM binding, insofar as it affects $V_{\mathrm{pk}}$, affects the relative energies of the vestigial transporter states of prestin. In light of this reasoning, generalizing to other SLC26 homologs becomes more understandable, because other homologs presumably have not merely vestigial but actual bona fide transporter states, e.g., outward and inward facing. When CaM binds these transporters, the relative energies of the conformational states would be perturbed, which would potentially result in alterations of transporter kinetics, electrogenicity, and/or rectification. For example, assuming the oft-invoked alternatingaccess transporter model, lowering the energy of one of the states through CaM binding might reduce the rate of conformational switching and hence transport activity. Although other SLC26 transporters are inhibited by intracellular calcium [SLC26A3 (Lamprecht et al., 2009; Keller et al., 2013) and SLC26A9 (Loriol 
et al., 2008)], consistent with the generality of the CaM interaction proposed here, it will be intriguing to see which of these functional consequences, if any, occur as a result of CaM binding to the other SLC26 transporters.

\section{References}

Alper SL, Sharma AK (2013) The SLC26 gene family of anion transporters and channels. Mol Aspects Med 34:494-515. CrossRef Medline

Ashmore J (2008) Cochlear outer hair cell motility. Physiol Rev 88:173-210. CrossRef Medline

Babu M, Greenblatt JF, Emili A, Strynadka NC, Reithmeier RA, Moraes TF (2010) Structure of a SLC26 anion transporter STAS domain in complex with acyl carrier protein: implications for E. coli YchM in fatty acid metabolism. Structure 18:1450-1462. CrossRef Medline

Bai JP, Navaratnam D, Samaranayake H, Santos-Sacchi J (2006) En block C-terminal charge cluster reversals in prestin (SLC26A5): effects on voltage-dependent electromechanical activity. Neurosci Lett 404:270275. CrossRef Medline

Brownell WE, Bader CR, Bertrand D, de Ribaupierre Y (1985) Evoked mechanical responses of isolated cochlear outer hair cells. Science 227:194196. CrossRef Medline

Chang MH, Plata C, Sindic A, Ranatunga WK, Chen AP, Zandi-Nejad K, Chan KW, Thompson J, Mount DB, Romero MF (2009) Slc26a9 is inhibited by the R-region of the cystic fibrosis transmembrane conductance regulator via the STAS domain. J Biol Chem 284:28306-28318. CrossRef Medline

Cheatham MA, Zheng J, Huynh KH, Du GG, Gao J, Zuo J, Navarrete E, Dallos P (2005) Cochlear function in mice with only one copy of the prestin gene. J Physiol 569:229-241. CrossRef Medline

Cheng J, Sweredoski MJ, Baldi P (2005) Accurate prediction of protein disordered regions by mining protein structure data. Data Min Knowl Disc 11:213-222. CrossRef

Dallos P (1992) The active cochlea. J Neurosci 12:4575-4585. Medline

Dallos P, He DZ, Lin X, Sziklai I, Mehta S, Evans BN (1997) Acetylcholine, outer hair cell electromotility, and the cochlear amplifier. J Neurosci 17: 2212-2226. Medline

Dorwart MR, Shcheynikov N, Baker JMR, Forman-Kay JD, Muallem S, Thomas PJ (2008) Congenital chloride-losing diarrhea causing mutations in the STAS domain result in misfolding and mistrafficking of SLC26A3. J Biol Chem 283:8711-8722. CrossRef Medline

Dosztányi Z, Csizmok V, Tompa P, Simon I (2005) IUPred: web server for the prediction of intrinsically unstructured regions of proteins based on estimated energy content. Bioinformatics 21:3433-3434. CrossRef Medline

Eybalin M (1993) Neurotransmitters and neuromodulators of the mammalian cochlea. Physiol Rev 73:309-373. Medline

Frolenkov GI, Mammano F, Belyantseva IA, Coling D, Kachar B (2000) Two distinct $\mathrm{Ca}^{2+}$-dependent signaling pathways regulate the motor output of cochlear outer hair cells. J Neurosci 20:5940-5948. Medline

Hallworth R, Stark K, Zholudeva L, Currall BB, Nichols MG (2013) The conserved tetrameric subunit stoichiometry of slc26 proteins. Microsc Microanal 19:799-807. CrossRef Medline

He DZ, Dallos P (1999) Somatic stiffness of cochlear outer hair cells is voltage-dependent. Proc Natl Acad Sci U S A 96:8223-8228. CrossRef Medline

He DZ, Jia S, Dallos P (2003) Prestin and the dynamic stiffness of cochlear outer hair cells. J Neurosci 23:9089-9096. Medline

Homma K, Dallos P (2011) Evidence that prestin has at least two voltagedependent steps. J Biol Chem 286:2297-2307. CrossRef Medline

Homma K, Miller KK, Anderson CT, Sengupta S, Du GG, Aguiñaga S, Cheatham M, Dallos P, Zheng J (2010) Interaction between CFTR and prestin (SLC26A5). Biochim Biophys Acta 1798:1029-1040. CrossRef Medline

Homma K, Duan C, Zheng J, Cheatham MA, Dallos P (2013) The V499G/ $\mathrm{Y} 501 \mathrm{H}$ mutation impairs fast motor kinetics of prestin and has significance for defining functional independence of individual prestin subunits. J Biol Chem 288:2452-2463. CrossRef Medline

Ikebe M, Kambara T, Stafford WF, Sata M, Katayama E, Ikebe R (1998) A hinge at the central helix of the regulatory light chain of myosin is critical for phosphorylation-dependent regulation of smooth muscle myosin motor activity. J Biol Chem 273:17702-17707. CrossRef Medline

Ishida T, Kinoshita K (2007) PrDOS: prediction of disordered protein regions from amino acid sequence. Nucleic Acids Res 35:W460-W464. CrossRef Medline
Keller JP, Homma K, Dallos P (2013) Pixels as ROIs (PAR): a less-biased and statistically powerful approach for gleaning functional information from image stacks. PLoS One 8:e69047. CrossRef Medline

Kere J (2006) Overview of the SLC26 family and associated diseases. Novartis Found Symp 273:2-11; discussion 11-18:261-264.

Ko S, Zeng W, Dorwart M, Luo X, Kim K, Millen L, Goto H, Naruse S, Soyombo A, Thomas P, Muallem S (2004) Gating of CFTR by the STAS domain of SLC26 transporters. Nat Cell Biol 6:343-350. CrossRef

Lamprecht G, Hsieh CJ, Lissner S, Nold L, Heil A, Gaco V, Schäfer J, Turner JR, Gregor M (2009) Intestinal anion exchanger down-regulated in adenoma (DRA) is inhibited by intracellular calcium. J Biol Chem 284: 19744-19753. CrossRef Medline

Linding R, Jensen LJ, Diella F, Bork P, Gibson TJ, Russell RB (2003) Protein disorder prediction: implications for structural proteomics. Structure 11: 1453-1459. CrossRef Medline

Loriol C, Dulong S, Avella M, Gabillat N, Boulukos K, Borgese F, Ehrenfeld J (2008) Characterization of SLC26A9, facilitation of $\mathrm{Cl}^{-}$transport by bicarbonate. Cell Physiol Biochem 22:15-30. CrossRef Medline

Mistrík P, Daudet N, Morandell K, Ashmore JF (2012) Mammalian prestin is a weak $\mathrm{Cl}^{-} / \mathrm{HCO}_{3}{ }^{-}$electrogenic antiporter. J Physiol 590:5597-5610. CrossRef Medline

O’Neil KT, DeGrado WF (1990) How calmodulin binds its targets: sequence independent recognition of amphiphilic alpha-helices. Trends Biochem Sciences 15:59-64. CrossRef

Pasqualetto E, Seydel A, Pellini A, Battistutta R (2008) Expression, purification and characterisation of the C-terminal STAS domain of the SLC26 anion transporter prestin. Protein Expr Purif 58:249-256. CrossRef Medline

Pasqualetto E, Aiello R, Gesiot L, Bonetto G, Bellanda M, Battistutta R (2010) Structure of the cytosolic portion of the motor protein prestin and functional role of the STAS domain in SLC26/SulP anion transporters. J Mol Biol 400:448-462. CrossRef Medline

Radivojac P, Vucetic S, O'Connor TR, Uversky VN, Obradovic Z, Dunker AK (2006) Calmodulin signaling: analysis and prediction of a disorderdependent molecular recognition. Proteins 63:398-410. CrossRef Medline

Radivojac P, Iakoucheva LM, Oldfield CJ, Obradovic Z, Uversky VN, Dunker AK (2007) Intrinsic disorder and functional proteomics. Biophys J 92: 1439-1456. CrossRef Medline

Rode B, Dirami T, Bakouh N, Rizk-Rabin M, Norez C, Lhuillier P, Lorès $\mathrm{P}$, Jollivet M, Melin P, Zvetkova I, Bienvenu T, Becq F, Planelles G, Edelman A, Gacon G, Touré A (2012) The testis anion transporter TAT1 (SLC26A8) physically and functionally interacts with the cystic fibrosis transmembrane conductance regulator channel: a potential role during sperm capacitation. Hum Mol Genet 21:1287-1298. CrossRef Medline

Santos-Sacchi J, Kakehata S, Takahashi S (1998) Effects of membrane potential on the voltage dependence of motility-related charge in outer hair cells of the guinea-pig. J Physiol 510:225-235. CrossRef Medline

Sharma AK, Ye L, Baer CE, Shanmugasundaram K, Alber T, Alper SL, Rigby AC (2011) Solution structure of the guanine nucleotide-binding STAS domain of SLC26-related SulP protein Rv1739c from Mycobacterium tuberculosis. J Biol Chem 286:8534-8544. CrossRef Medline

Shen X, Valencia CA, Szostak J, Dong B, Liu R (2005) Scanning the human proteome for calmodulin-binding proteins. Proc Natl Acad Sci U S A 102:5969-5974. CrossRef Medline

Slepecky NB, Ulfendahl M (1993) Evidence for calcium-binding proteins and calcium-dependent regulatory proteins in sensory cells of the organ of Corti. Hear Res 70:73-84. CrossRef Medline

Uversky VN, Dunker AK (2010) Understanding protein non-folding. Biochim Biophys Acta 1804:1231-1264. CrossRef Medline

Ward JJ, Sodhi JS, McGuffin LJ, Buxton BF, Jones DT (2004) Prediction and functional analysis of native disorder in proteins from the three kingdoms of life. J Mol Biol 337:635-645. CrossRef Medline

Xue BB, Dunbrack RLR, Williams RWR, Dunker AKA, Uversky VNV (2010) PONDR-FIT: A meta-predictor of intrinsically disordered amino acids. Biochim Biophys Acta 1804:996-1010. CrossRef Medline

Zheng J, Shen W, He DZ, Long KB, Madison LD, Dallos P (2000) Prestin is the motor protein of cochlear outer hair cells. Nature 405:149-155. CrossRef Medline

Zheng J, Du GG, Matsuda K, Orem A, Aguiñaga S, Deák L, Navarrete E, Madison LD, Dallos P (2005) The C-terminus of prestin influences nonlinear capacitance and plasma membrane targeting. J Cell Sci 118:29872996. CrossRef Medline 